\title{
El estudio de la puesta en acto de las políticas educativas en clave microfísica
}

\author{
The study of the enactment of educational policies in a microphysical key
}

\author{
Carla A. Villagran \\ carla_villagran@hotmail.com \\ CIT Golfo San Jorge-CONICET. Universidad \\ Nacional de la Patagonia Austral, Argentina
}

Cita sugerida: Villagran, C. A. (2020). El estudio de la puesta en acto de las políticas educativas en clave microfísica. Revista Latinoamericana de Metodología de las Ciencias Sociales, 10(2), e079. https://doi.org/10.24215/18537863e079

Recepción: 23 Marzo 2020

Aprobación: 10 Septiembre 2020

Publicación: 01 Diciembre 2020

\begin{abstract}
Resumen: En este artículo desarrollamos algunas problematizaciones y reflexiones en torno a la construcción metodológica y conceptual para el estudio de la puesta en acto (Ball, Maguire \& Braun, 2012) de políticas educativas a nivel de las instituciones escolares. Proponemos un abordaje etnográfico (Rockwell, 1987, 1995) y una analítica microfísica (Deleuze, 2014) de los procesos de puesta en acto, centrados en la descripción de los efectos y modos de afectación que producen las políticas una vez que llegan a las escuelas. Partimos de la premisa de que no estudiamos las escuelas sino "en" escuelas y atendiendo a los saberes y experiencias de los sujetos.

Palabras clave: Abordaje microfísico, Etnografía, Políticas educativas, Puesta en acto.

Abstract: In this article we develop some problems and reflections on the methodological and conceptual construction for the study of the enactment (Ball, Maguire \& Braun, 2012) of educational policies at the level of school institutions. We propose an ethnographic approach (Rockwell, 1987, 1995) and a microphysical analytic (Deleuze, 2014) of the enactment processes focused on the description of the effects and modes of affectation that the policies produce once they reach the schools. We start from the premise that we do not study in schools but "in" schools and attending to the knowledge and experiences of the subjects.
\end{abstract}

Keywords: Microphysical approach, Ethnography, Educational policies, Enactment.

\section{INTRODUCCIÓN}

Abordamos la construcción de la metodología entendiendo que se trata del camino o el plan trazado en una investigación para la consecución de los objetivos propuestos, en vinculación con el problema de investigación y la construcción conceptual. La experiencia investigativa nos viene mostrando que esa construcción metodológica es un camino que se parece más a la rusticidad de la tierra que a la rectitud del cemento. Así, el camino metodológico suele por momentos encontrar piedras, devenir llanuras, desplazamientos, redefiniciones de ruta y la generación de caminos alternativos para la construcción del objeto de estudio. 
La construcción conceptual constituye una caja de herramientas (Foucault, 1992), una guía que no constriñe por anticipado la situación problemática sino que posibilita su descripción y comprensión. En este sentido, el ensamblado conceptual, para la descripción de los procesos de interpretación y traducción de las políticas educativas (Ball, Maguire \& Braun, 2012) en escuelas de educación secundaria, incorpora la perspectiva foucaulteana, los estudios de la gubernamentalidad y de los afectos políticos ${ }^{1}$ (Ahmed, 2010; Berlant, 2011; Massumi, 2009).

En nuestra propuesta, centralmente etnográfica, se vuelve necesario pensar cuál es la posición de las y los investigadores respecto de las situaciones problemáticas. El problema de investigación resulta ese pasar de algo que nos inquieta y que, al principio, suele ser indefinido, a una elaboración que sea producto de hacernos preguntas respecto de procesos sociales, en este caso educativos. De allí que el objeto de estudio sea el resultado de ese proceso de inteligibilidad y construcción.

A partir de lo expuesto, se propone un conjunto de preguntas que orientan la reflexión para pensar la investigación en las instituciones escolares más allá de la casuística. En el recorrido propuesto, se avanzará simultáneamente en dos sentidos: por un lado, explicitando nociones conceptuales y estrategias metodológicas, y por otro, realizando el ejercicio, no sencillo, de reflexionar sobre la propia tarea de investigación. Así, el artículo se organiza en tres ejes: a) los aportes y posibilidades del abordaje microfísico en el estudio de las políticas educativas; b) la analítica de las prácticas políticas en las instituciones y las situaciones coyunturales; y c) la descripción de las prácticas escolares en escala cotidiana y el posicionamiento político de les investigadores.

\section{Construcción CONCEPTUAL Y METOdológica PARA EL ESTUdio DE LAS POLÍticas EDUCATIVAS}

Este primer eje de problematización se vincula, por un lado, con describir brevemente la construcción metodológica y conceptual que orienta las investigaciones que venimos realizando y, por otro, con exponer algunos aportes y potencialidades para el estudio de la vida escolar de las políticas educativas. La intersección de la sociología política de la educación y los estudios del currículum constituye el terreno en el que se anclan los aportes que aquí ponemos en discusión. En ese cruce de debates conceptuales y de tradiciones de investigación tiene lugar la pregunta política por las reformas, ${ }^{2}$ sus legados e impactos en la vida de las escuelas, que está adquiriendo cada vez más relevancia (Cantero et al., 2006; Feldfeber y Gluz, 2011; Ferreyra, 2012; Frigerio, 2000; Giovine, 2008, 2012; Gorostiaga y Tello, 2011; Miranda, 2001, 2017; Tiramonti 2015a, 2015b).

Tal como Foucault (1992) lo dijo, si pensamos la construcción conceptual del objeto como caja de herramientas, estamos refiriendo a aquello que envuelve reflexiones, relaciones, tiempos, así como la inscripción en un aquí y ahora. Pensar la construcción conceptual o la práctica de construcción y conceptualización en una investigación como caja de herramientas involucra partir de la disponibilidad de instrumentos que están "a la mano" pero que merecen un tratamiento coherente e integrado a la construcción del objeto de estudio. En este sentido, el asunto radica en los usos que hacemos de las perspectivas, de los conceptos, con qué otros conceptos y/o líneas temáticas los combinamos y cómo lo hacemos. Eso es, justamente, de lo que hablaba Foucault en sus escritos: de coherencia en los abordajes y también en la tarea analítica.

Foucault señala en La arqueología del saber que su trabajo cava fosas para definir su propio camino. Ésa podría ser la pista de las pistas cuando "usamos" perspectivas, conceptualizaciones, ideas o planteos de diversa autoría. Esto es, reconocer que siempre hay un suelo sobre el que reposan nuestros análisis, que son puntos de partida y aportes fundamentales que debemos reconocer, pero que en definitiva el camino lo vamos haciendo nosotros, permanentemente, con lo artesanal y creativo que tiene la investigación. 
En el marco de estos planteos, la unidad de análisis fueron escuelas secundarias públicas del norte de la provincia de Santa Cruz. Siguiendo a Goetz y LeCompte (1988), la selección de las escuelas se orientó principalmente por el criterio de su carácter público y por un criterio de fragmentación urbana y socioeducativa, que son producto de la utilización de la georreferenciación. La selección basada en criterios se combinó en el análisis con la comparación constante de datos. La estrategia de la comparación constante implica un análisis sistemático de incidentes con la finalidad de generar teoría a partir de similitudes, diferencias y regularidades en los procesos.

La obtención de información se realizó mediante la observación participante y la entrevista en profundidad (Achilli, 2005; Guber, 2005) o entrevista etnográfica (Ameigeiras, 2007) a miembros de los equipos directivos, asesores pedagógicos y docentes de las escuelas seleccionadas. A su vez, se realizaron entrevistas flash a los sujetos en las escuelas en los encuentros de menor duración. Las entrevistas se definieron siempre como "conversaciones amistosas en las que el investigador introduce lentamente nuevos elementos" (Spradley, 1979, en Ameigeiras, 2007). En este sentido, se procuró sostener la mirada y escucha atendiendo a los gestos, silencios, esquivos, entre otros aspectos constitutivos del contexto de la entrevista. La entrevista definida como entrevista en profundidad o entrevista etnográfica es una herramienta metodológica para profundizar en los significados y puntos de vista de los sujetos entrevistados. De manera que elaboramos diferentes protocolos de entrevistas, que contenían temas y subtemas orientadores más que preguntas a realizar, con algunas variaciones según se tratase de docentes, asesores o rectores de las escuelas secundarias. Así, las entrevistas resultaron de la construcción de un diálogo en torno a la problematización propuesta por la investigadora pero que incorporó también las preocupaciones de los entrevistados. En cuanto a la observación participante y el registro de campo, se relacionan a partir de la herramienta fundamental del trabajo antropológico que es la descripción densa (Geertz, 2000).

Asimismo, como parte del trabajo de campo, que se extendió entre los años 2013 y 2017, se realizó un proceso de rastreo e identificación de documentos, textos y/o materiales oficiales. Los documentos relevados fueron analizados en términos de qué aspectos y/o temas son los más enfatizados, cuáles son los planteos más incidentes, qué conjunto de conceptos son los más recurrentes y qué lineamientos para las escuelas cobran mayor relevancia. Si bien se realizó este proceso de reconocimiento de los textos que fueron recibidos por las escuelas, no fue el objetivo realizar un análisis de correlación entre éstos y su puesta en acción.

Así, en la construcción metodológica proponemos el análisis de los enunciados en el sentido que Foucault indica, sin intentos de bucear en los significados ocultos o reprimidos, ni tampoco con ánimos de volcarse a la imposible tarea de descifrar lo que "se quiso decir", recuperando la materialidad marcada por coordenadas espaciales y temporales atendiendo a lo que "se dijo". Ello se aplica tanto al análisis de los textos políticos como al de los textos de descripción densa producto del registro en las observaciones participantes realizadas y de los textos de las entrevistas.

La información obtenida fue organizada y sistematizada cualitativamente a partir de la construcción de categorías analíticas medianteel software de análisis de datos cualitativos Atlas Ti. El proceso de categorización se realizó a partir de la codificación abierta y la codificación axial. La primera refiere a una primera selección de fragmentos de material empírico y la definición de categorías iniciales. Luego, la segunda instancia supone la identificación de categorías centrales que constituyen los ejes del análisis. Esta dinámica de categorización puede reiterarse varias veces hasta que el investigador considera que cumple con sus criterios.

En los puntos que siguen, se despliegan las implicancias y problematizaciones del abordaje metodológico propuesto para la analítica de los procesos de puesta en acto de políticas educativas. 


\subsection{El abordaje de la reforma en clave microfísica}

Desde el inicio del proceso de investigación, pasando por el planteamiento del problema, el trabajo de campo y el trabajo analítico para devenir en la construcción del objeto de estudio, nuestra perspectiva ha procurado avanzar sosteniendo los siguientes ejes:

a) el análisis de la reforma en clave microfísica, más allá de las oposiciones macro y micro políticas;

b) el estudio de la reforma atendiendo a la perspectiva genealógica como rupturas y continuidades (entre lo viejo y lo nuevo);

c) el estudio de las políticas más allá de la dicotomía entre lo escrito y lo real, y su comprensión como prácticas discursivas y no discursivas que involucran momentos que hacen al currículum escolar;

d) el análisis de los enunciados en su superficie y materialidad.

En este sentido, un aspecto metodológico central que guía el proceso de investigación es entender que las políticas educativas en su puesta en acto y devenir no constituyen desviaciones de la norma, sino que se trata del hecho de que los sujetos la interpreten, se apropien, resistan, moldeen, entre otras cuestiones, tanto a partir de lo que ya saben hacer como de las condiciones institucionales y laborales en las que tienen lugar. Es decir, la puesta en acto no se trata de desviaciones de la ley o de las políticas sino que, desde una lectura microfísica de las políticas, ellas contienen, más que prohibiciones o impedimentos, habilitaciones a las más variadas formas de interpretación y materialización. Al decir de Deleuze (2014), la ley o las leyes no pueden separarse de los casos y sus condiciones de aplicación:

(...) una microfísica de la ley es un punto de vista en el cual la ley ya no es separable de sus decretos de aplicación y ya no es separable de sus casos de interpretación. No hay ley que no suscite problemas de interpretación (p. 63).

De allí que proponemos un estudio en esta clave, atendiendo a que la política educativa continúa en la escuela a la hora de interpretar y traducir. Asimismo, las interpretaciones y formas de traducción obedecen más a multiplicidades que a binarismos, a complementariedades más que a oposiciones.

Mirar la escuela, las relaciones de poder desde una perspectiva microfísica, posibilita aproximarnos a la comprensión de las políticas educativas desde/en la vida de la escuela, con la mirada centrada en las condiciones reales de funcionamiento y en las dinámicas cotidianas. Se trata de intentar agarrar al poder “(...) en sus extremidades, en sus confines últimos, allí donde se vuelve capilar, de asirlo en sus formas e instituciones más regionales, más locales (...)" (Foucault, 1992, p. 144).

Un abordaje microfísico ${ }^{3}$ supone el pasaje de lo macro a lo micro, pero no entendiendo que lo micro es una expresión en menor tamaño de lo macro; no es una miniaturización (Deleuze, 2014). Foucault insiste en que lo macro y lo micro no se oponen y que lo micro no es expresión en pequeño de lo macro, sino que son aspectos que obedecen a naturalezas diferentes. Mientras lo macro es compatible con estructuras molares, lo micro se caracteriza por lo molecular, por lo infinitesimal, por los intersticios que tienen lugar en un nivel micropolítico de las relaciones de poder. De ello dio cuenta en las oportunidades en las que dedicó su atención a describir las precauciones del método. Así, en Historia de la sexualidad expone: "Ningún 'foco local', ningún 'esquema de trasformación' podría funcionar sin inscribirse al fin y al cabo, por una serie de encadenamientos sucesivos, en una estrategia de conjunto" (Foucault, 2008, p. 122). Las relaciones de poder, las microrelaciones, las relaciones moleculares, se inscriben siempre en formas globales, no como homogeneización ni representación a otra escala sino como niveles de diferente naturaleza.

Poder avanzar en la recuperación de ese nivel molecular, en la inteligibilidad de la historia (Foucault, 1992), en el detalle de su complejidad y multiplicidad es sin duda la tarea metodológica central, que requiere permanentemente reflexión epistemológica y evaluación de rigurosidad.

La clave microfísica nos ha posibilitado avanzar en la descripción de las prácticas de puesta en acto de una reforma a nivel de las escuelas, recuperando las experiencias, expectativas, sentires y modos de interpretación 
de los sujetos. Ello, a la vez que caracterizar los afectos políticos producidos por las políticas y las estrategias y saberes puestos en funcionamiento para la materialización de las transformaciones.

\subsection{El enfoque etnográfico y sus aportes en el diseño cualitativo de la investigación}

En el punto precedente dimos cuenta de las implicancias del estudio de los procesos de puesta en acto en clave microfísica. Ello, como abordaje metodológico, supone el ensamblado con el enfoque etnográfico (Batallán, 2007; Pallma y Sinisi, 2004; Rockwell, 1987, 1995; Rockwell y Anderson-Levitt, 2015; Rockwell y Ezpeleta, 2007) para la documentación de las prácticas de interpretación y traducción de la política (Ball, Maguire \& Braun, 2012).

Las tradiciones más antiguas de la antropología de la educación se remontan al año 1950 en Alemania, EE.UU., Japón, Brasil y México. Los estudios etnográficos del aprendizaje o de la educación surgieron luego de la década de 1970, o más adelante, en países como Francia, Israel, Hungría, China y Guinea (AndersonLevitt, 2012). Hoy, estos enfoques se alejan de esos inicios de la etnografía como estudio de las culturas o pueblos lejanos (Pallma y Sinisi, 2004) y advierten una pluralidad de perspectivas que atienden a un trabajo de campo situado en los propios espacios de los equipos de investigación.

Los aportes del enfoque etnográfico radican en su carácter abierto. Si bien la investigación parte de un conjunto de problemas, preguntas y categorías, posibilita en todo el proceso ir incorporando aspectos que resulten significativos y que surjan en el trabajo de campo. De modo que el proceso etnográfico se vuelve transitivo y recursivo, además de ser ya profundamente reflexivo (Marcus, 2013).

Uno de los aportes de este enfoque consiste en capturar la mirada de los sujetos, en poder registrar y hacer inteligibles experiencias que acontecen en la urdimbre que resulta la cotidianeidad escolar. Urdimbre en la que quien investiga puede "meterse" para describir y comprender los aspectos en detalle de las relaciones de poder microfísicas; tarea que no podría realizarse si no es a través de la confianza construida con los sujetos de la investigación. Por ello, sostenemos que esta perspectiva aporta al conocimiento de esos detalles que se vuelven esenciales en la vida diaria y que serían difícilmente asequibles desde otros enfoques. Incluso, entendemos que la etnografía es aquello que Youdell (2010), desde una perspectiva postestructuralista, denomina como el lugar del detalle. Ese lugar del detalle de la vida de la escuela que involucra una renuncia a lo universal y una apuesta por la singularidad. Sostenemos que la singularidad queda definida en el acontecimiento y el caso, y no en el individuo (Grinberg, 2008; Villagran, 2016).

Ahora bien, sabemos que esa documentación, descripción y construcción de conocimiento siempre ocurre como parte de las propias definiciones del diseño de investigación y de las limitaciones de los sujetos que investigan. No aspiramos a la pretensión malinowskiana de la descripción densa de la totalidad de los aspectos sociales, culturales y psicológicos (Montes de Oca, 2015). En cambio pretendemos, sí, una descripción densa en detalles que recupere lo dicho por los sujetos, situada en la serie espacio-tiempo, y un análisis que procuró focalizarse en las prácticas y relaciones de poder y sus efectos en escuelas secundarias.

\subsection{Perspectiva cíclica y puesta en acto de las políticas desde la sociología política de la educación}

Entendemos que las temporalidades de las políticas, en este caso de reforma, se combinan y enlazan tanto en la inmediatez como en el largo plazo. De esta manera, esta investigación se ha propuesto sostener un fuerte acento en la sincronía, es decir, la realización de trabajo de campo desde el momento de llegada de la reforma a la escuela, como también recuperar las prácticas de materialización en el devenir. En este punto, la incorporación de la perspectiva cíclica, propuesta por Bowe, Ball \& Gold (1992), constituye una mirada conceptual y metodológica pero también es resultado del trabajo en terreno. 
En el marco de la sociología política de la educación, el ciclo de las políticas constituye una herramienta conceptual y heurística para el estudio de cómo estas llegan a las escuelas y qué efectos producen en su trayectoria (Miranda, 2001; Miranda y Lamfri, 2017; Barbosa Moreira, 1990). Esta noción rompe con los análisis lineales y rígidos de las políticas educativas, superando los planteos vinculados a la implementación y/o ejecución de los lineamientos políticos a cargo de los sujetos. Ball (2009) explicita que esta perspectiva cíclica no es una descripción de la política en sí, sino que es una manera de pensar cómo se hacen las políticas. Sintéticamente, es una manera de investigar y teorizar sociológicamente acerca de las políticas educativas. En el caso que nos convoca, nos enfocamos en ese momento o contexto en el que la política llega a la escuela y es interpretada y materializada por los sujetos. Un momento que no acusa finalización sino que se extiende en una larga duración.

Asimismo, en desarrollos posteriores Ball y su equipo avanzan en la complejización de esta perspectiva a partir de la noción de policy enactment. Esta idea, vinculada a la teoría de la política en acción, supone comprender que las políticas son interpretadas y materializadas de diferentes y variadas formas en las escuelas por sujetos con una historia y unas experiencias. La noción de policy enactment, es decir, de la puesta en acto (enactment) o en acción de una política, abre un campo de análisis que se focaliza en los aspectos materiales y subjetivos de la interpretación y traducción de las políticas. De manera que, aunque comporta una instancia de características diferenciales, la puesta en acto en las escuelas expresa también prácticas políticas de producción. Las dinámicas que se generan en las escuelas en torno a la traducción de las políticas encuentran su soporte en la materialidad escolar (Dussel, 2019a, 2019b; Gleason, 2017; Lawn y Grosvenor, 1999, 2001) y se transforman en un aspecto central de esa puesta en acción. Además de ello, se ensamblan con las posibilidades concretas de disponibilidad del cuerpo docente para poner en acto los lineamientos. En este proceso de actuación de la política, constantemente las escuelas tienen que tomar decisiones, a veces dolorosas, sobre dónde se encuentran sus prioridades políticas.

Recuperamos los aportes de Ball, cuya base es foucaulteana, y proponemos un estudio en clave microfísica que se acerque a la complejidad de la vida escolar pero, especialmente, a la vida de los sujetos que hacen la escuela cotidianamente, analizando los efectos que producen las tecnologías de regulación puestas en juego en tiempos de una reforma en las subjetividades.

\section{LA ANALÍTICA DE LAS PRÁCTICAS POLÍTICAS Y LAS SITUACIONES COYUNTURALES EN EL PROCESO DE OBTENCIÓN DE LA INFORMACIÓN}

En el diseño de investigación que construimos, siempre está presente la pregunta en torno a qué reflexividad metodológica conduce por mejor camino. Como expusimos, las acciones metodológicas se asientan en la creatividad y el pensamiento de los equipos de investigación. Así, y siguiendo a Youdell (2010), la inteligibilidad de nuestros propios procesos es una columna que vertebra el trabajo de campo y la construcción categorial. En este sentido, aquí nos enfocaremos en algunas preguntas y reflexiones en torno al trabajo de campo y a la analítica de las prácticas políticas en las instituciones escolares, y qu sucede cuando las situaciones coyunturales adquieren protagonismo. Asimismo, y vinculado a lo anterior, reflexionamos en torno a cómo no enfatizar el pesimismo de los sujetos a travs de la investigación y cómo describir sin romantizar la escuela y lo que allí acontece.

El equipo de investigación viene trabajando en educación secundaria desde el año 2013 en la provincia de Santa Cruz. Algunos de los conflictos que afectan a las instituciones escolares son de público conocimiento por su circulación en los medios masivos de comunicación. Entre esos conflictos pueden mencionarse aquellos vinculados a reclamos salariales docentes y otros referidos a condiciones de trabajo del personal de maestranza y auxiliares de educación, falta de agua y de calefacción, entre los más recurrentes. Por momentos, este escenario constituye un telón de fondo que es móvil y admite variaciones; sin embargo, la profundidad y los niveles de afectación que generan, producen una especie de enredo entre las bambalinas. Apuntamos 
aquí a dar cuenta de cómo algunas situaciones coyunturales adquieren protagonismo, por su actualidad, por su relevancia, por su nivel de afectación, y de cómo hemos avanzado en la obtención de la información y en su analítica. Cuestión sensible cuando se trabaja en enfoques etnográficos, en la recuperación del detalle de la cotidianidad de la puesta en acto de políticas.

En este punto, probablemente, se encuentre uno de los actos metodológicos más difíciles — pero nodales - de realizar en la investigación, que es elaborar la "cintura" a partir de la reflexividad. ¿Cómo no quedarnos enredados en las bambalinas en las que también están los sujetos en la escuela? Ello, además, porque las situaciones que ocurren en la escuela, o que ocurren en sus bordes y que la afectan, o que ocurren "arriba" y embisten ese "abajo", son más que una escenografía que se cambia. Existe y con él nos venimos encontrando en estos años: un clima de malestar en las escuelas que, si la observancia epistemológica y metodológica no es rigurosa, pasa a primer plano, sesgando otros aspectos. ${ }^{4}$

El ejercicio de construcción categorial, que es permanente, contribuye justamente a armar el rompecabezas del trabajo de campo y a ubicar las capas que componen la producción de significados de los sujetos, como también el lugar que ocupamos nosotros en la escena. La producción textual, en el enfoque etnográfico, permite objetivar tanto la información como los propios sentires, no para tomar distancia y sostener cierta asepsia sino para tomar ese tiempo lógico que requiere sostener la mirada, comprender y concluir. ${ }^{5}$

Estos ejercicios metodológicos se vuelven especialmente fundamentales cuando investigamos en educación secundaria que, en los últimos años, fue uno de los niveles con mayor concentración de políticas de reforma en su estructura, currículum, organización pedagógica y régimen académico. Hay un oleaje intempestivo que es vivido por los sujetos con mucho malestar, que queda circulando en el espacio y tiempo escolar como malestar en sí (Villagran, 2018; Villagran y Grinberg, 2019). Parte de los procesos de responsabilización que generan las políticas, en su puesta en acto y devenir, intensifican la tarea educativa además del malestar. Aquí también, y y vinculado a lo que venimos exponiendo, nos preguntamos cómo no enfatizar el pesimismo de los sujetos a través de la investigación y cómo describir la escuela y las prácticas de los sujetos sin romantizar. Ello, cuando la propuesta de estudiar las políticas educativas desde la escuela involucra esa hechura sociopolítica que tiene lugar en los tiempos y espacios escolares. Más aún porque investigaciones de estas características se encaminan en las sendas de lo cotidiano, en los intersticios y en las prácticas anónimas que arquitecturan la experiencia escolar.

\section{LA DESCRIPCIÓN DE LAS PRÁCTICAS ESCOLARES EN ESCALA COTIDIANA Y CÓMO ELLO SE ENSAMBLA CON EL POSICIONAMIENTO POLÍTICO DE LOS INVESTIGADORES}

El tipo de estudio que proponemos, en línea con los planteos recién expuestos, no pretende aportar a la representatividad estadística sino avanzar en la recuperación de las vivencias y experiencias de los sujetos, aquello que Foucault (2002) explicaba como "dibujar configuraciones singulares". Sin embargo, el trabajo con colegas, con otros equipos, el rastreo de las investigaciones que se están realizando actualmente o hace algunos aos indica que más allá de la historicidad particular de los casos en estudio hay una vinculación con otras historicidades diversas. En este punto, creemos que la caracterización de las prácticas escolares, de las transformaciones en los dispositivos pedagógicos, en los procesos de subjetivación encuentra en la comparación no un efecto unificador sino uno multiplicador. De allí que sostenemos que estudios de estas características pueden aportar a recuperar los efectos a nivel de las instituciones de las formas institucionales más amplias y de la producción sociopolítica en sí.

El posicionamiento político es inmanente al posicionamiento metodológico. Así, el abordaje que proponemos parte de y se sostiene en una doble preocupación. Por un lado, la producción de conocimiento científico y, por otro, el compromiso y la lucha por condiciones más justas e igualitarias en lo social y educativo. El enfoque etnográfico en la investigación educativa supone que las investigadoras e investigadores se reconozcan a sí mismos como sujetos cuya existencia puede estar signada por las mismas condiciones de 
vida de los sujetos de la investigación. Esto es, aunque no siempre, compartir un mismo espacio social, con un conjunto de preocupaciones y problemáticas que también pueden ser comunes. Por ello, es central ser capaces de vernos en ese espacio social, ver las acciones que allí desarrollamos y que ello esté "dentro del foco de investigación y explotar sistemáticamente nuestra participación como investigadores en el mundo que estamos estudiando" (Hammersley y Atkinson, 1994, p. 20).

Se avanza aprendiendo a elaborar objetivos, a combinar técnicas, a categorizar e interpretar y construir datos, al tiempo que nos detenemos a pensar cómo lo estamos haciendo o si sería mejor hacerlo de otra manera. Nos detenemos porque sencillamente el sujeto tiene que procesar lo que hace, necesita tiempos para confiar en que las decisiones tomadas son las más coherentes, para ver si el proceder analítico es riguroso, entre otros aspectos. Así, detenerse a pensar, reflexionar y continuar o modificar la estrategia son inmanentes al trabajo investigativo. Esto es aquello que Youdell (2010) explica como la demanda del sujeto por la inteligibilidad. No sólo del objeto de estudio en construcción, sino de nosotros mismos como investigadores. Es la intención de volver inteligibles también las propias prácticas desenvueltas en el curso del trabajo de investigación.

En este sentido y, tal como sostiene Ameigeiras (2007), la práctica de investigación, en etnografía, transforma al investigador tanto en el proceso de construcción social del conocimiento como en la conformación de una experiencia vital irremplazable en el trabajo de campo. Los interrogantes que al inicio de trabajo de campo fueron apareciendo se vincularon a cómo nos recibirían en las escuelas, hasta qué punto podría resultar incómoda nuestra presencia, cómo hacer para no cometer errores que costaran el cierre de puertas. Algunos de estos interrogantes, vinculados, por supuesto, a las ansiedades propias de la tarea, continuaron durante todo el trabajo de campo y otros fueron disipándose. La sola presencia de quienes investigan en el campo modifica las actuaciones. Esto es algo que no podría negarse y que, de hecho, no intentamos negar, sino que sabemos que nuestra presencia implicó e implica sensaciones y conductas distintas de las habituales por ser ajenos a ellas. Asimismo, es cierto que, a medida que nuestra presencia se hace más regular, también se vuelve un poco menos ajena. A la inversa, en la medida en que nuestra frecuencia en la escuela y en los barrios se fue incrementando, también nos pareció menos amenazante e inseguro ese espacio. Nuestras sensaciones y sentires también se fueron re-configurando. Ello, especialmente, cuando se refiere al posicionamiento del investigador en la escena. Como lo indica Foucault (2002) en su referencia a Las meninas de Velázquez: el autor forma parte del cuadro. De hecho, esa analítica de Foucault dirige la atención hacia el intercambio de miradas entre los sujetos; quienes investigamos no estamos fuera del cuadro que describimos y que proponemos comprender, sino que somos parte clave de él.

Como señala Rockwell (1987), la interacción etnográfica, por ser social, está fuera de nuestro control. El estar ahí, en ese momento, genera significados, interpretaciones, sensaciones, angustias en los sujetos. Sentirse observado condiciona y en ese sentido son centrales las miradas que los sujetos tienen de nosotras, cómo nos ubican en su cotidianeidad. Esas miradas forman parte integral del trabajo de campo (Rockwell, 1987).

Como investigadores, siempre tratamos de estar, de ver, de participar, de conversar, de que nos vean. Intención que recurrentemente estuvo acompañada por preguntas acerca de qué les pasa a las y los docentes cuando ven a estudiantes y a directivos y, a la inversa, qué les pasa cuando sienten que se les observa. Es decir, qué lectura hacen de nuestra presencia, de nuestras preguntas, de nuestra insistencia.

El qué se hará con la información, quién la verá, por dónde circulará es una de las grandes preocupaciones que encontramos al entrevistar en las escuelas. Este aspecto lo han manifestado tanto docentes como directivos; sin embargo, la preocupación no era que la información fuera conocida por la "gente de la universidad", sino por sus superiores, ya sean los directivos de la escuela, en el caso de los docentes, o las agencias políticas o de supervisión, en el caso de las rectoras y rectores. Así, lo dicho ya es, de por sí, una información comprometedora que podría retornar negativamente sobre los sujetos, tomando en cuenta la relación conflictiva con las agencias de gobierno provincial y local y lo que eso genera. 
En suma, tal como exponen Denzin \& Lincoln (2005), la tarea de investigación puede definirse como el oficio del "bricoleur o tejedor de colchas". Tejer, destejer, un punto que se escapa, un cálculo que no sale, una trama jaspeada, la impronta de la creatividad en el producto. Investigando, desplegamos y conjugamos diferentes estrategias para acercarnos al problema y construir el objeto de estudio. Pensamos la investigación como una práctica abierta en la que se pueden ir tomando decisiones, considerando los escenarios y sujetos de la investigación. En otras palabras, el oficio de la investigación, intrínsecamente, anida la rigurosidad del conocimiento científico con la creatividad y la imaginación.

\section{Conclusiones}

Este artículo tuvo como propósito exponer algunas conceptualizaciones, problematizaciones y reflexiones que orientaron una investigación en torno a la puesta en acto (Ball, Maguire \& Braun, 2012) de políticas de reforma a nivel de las instituciones educativas. Parte de esa problematización es dar cuenta y explicitar nuestras propias demandas por la inteligibilidad (Youdell, 2010). Es decir, realizar el ejercicio metodológico de objetivar nuestras propias prácticas de construcción conceptual y metodológica al mismo tiempo que mirar en detalle los procesos de obtención y análisis de la información.

La perspectiva elaborada se asienta en un ensamblado conceptual y metodológico configurado por un enfoque sociopolítico y microfísico de estudio de las políticas educativas, por un diseño de investigación cualitativo centrado fuertemente en la etnografía educativa y por una analítica de los enunciados en su inscripción temporal y espacial. En este planteo, los hilos conductores del estudio de los procesos de materialización de la política han sido la recuperación de los detalles y de las vivencias de los sujetos en torno a esos procesos y la lectura de las interpretaciones en sentido microfísico (Deleuze, 2014) como modalidades múltiples de lectura y apropiación y no como desviaciones de la norma.

Gran parte de la tarea como investigadoras e investigadores es poder mirar en ese detalle de las relaciones de poder, de los efectos y los afectos para reconstruir los procesos de interpretación y traducción de las políticas educativas. Así, como señalaFoucault, hay aspectos que sólo aparecen en la filigrana del trabajo analítico de los enunciados y las prácticas. Allí nos encontramos con que la afectividad es parte constitutiva de la puesta en acto de las políticas o un engranaje clave, que las configuraciones de las temporalidades y las espacialidades no son un mero dato en esa puesta en acto y que más allá del malestar hay fuerza de trabajo y voluntades que traccionan diariamente el sostenimiento de la escolaridad. Aquello que Berlant (2011) llama "optimismo cruel".

Tal vez, este último aspecto acerca del sostenimiento de la escolaridad es uno de los que ocupa más tiempo, energías y definiciones puesto que revalorizamos el lugar de la escuela en la trasmisión de la cultura y en los procesos de inclusión y contención social. He aquí otro de los recaudos cuando se tensiona la empatía por aquello que los sujetos narran, incluso de manera catártica, tocando las fibras más humanas en el trabajo de campo.

La capacidad de asombro es difícil de superar e, insistimos, los casos no dejan de conmovernos y de conducir el pensamiento a la ponderación de todas aquellas acciones que sostienen la educación pública, y a los adolescentes y jóvenes particularmente. No podemos dejar de conmovernos, de asombrarnos, de sentir. Pero tampoco podemos, en la tarea de producir conocimientos, hablar a través de otros o hacer denunciar a los otros lo deseable ante situaciones de desigualdad. En las escuelas en las que venimos realizando trabajo de campo nos encontramos con docentes, equipos directivos que buscan a padres y estudiantes, que gestionan recursos y disfrazan los gastos, que sacan la caja de herramientas del auto y arman bibliotecas, que organizan ferias de ropa y comida para gastos internos y compra de equipamiento, que sueñan, proyectan y plasman en planos cómo quisieran que sea su escuela, por mencionar solo algunas acciones. Por supuesto, ello nos condujo a reflexionar en torno a "lo que puede la escuela" o, en términos de Biesta y Säfström (2018), cuánta educación se puede brindar aún en las instituciones educativas. Esto es, describir las posibilidades que ofrece la escuela 
y cómo los docentes, directivos y asesores pedagógicos estructuran un esquema de acción especialmente pensado para los jóvenes y sus trayectorias escolares.

\section{ReFERENCIAS}

Abu-Lughod, L. (1988). Fieldwork of a Dutifuld Daughter. En S. Altorki y C. Fawzi (Eds.). Arab Woman in the field. Studying you own society (139-161). New York: Syracuse University Press.

Achilli, E. (2005). Investigar en antropología social. Los desafios de transmitir un oficio. Rosario: Laborde Editor.

Ahmed, S. (2010). Happy objects. En M. Gregg \& J. Seigworth (Eds.). The affect theory reader (pp. 29-51). Durham \& London: Duke University Press.

Ameigeiras, A. (2007). El abordaje etnográfico en la investigación social. En I. Vasilachis de Gialdino (Coord.), Estrategias de investigación cualitativa (pp. 107-149). Buenos Aires, Argentina: Gedisa.

Anderson-Levitt, K. M. (2012). Introduction: anthropologies and ethnographies of education worldwide. En Anthropologies of Education: A Global Guide to Ethnographic Studies of Learning and Schooling (pp. 1-28). New York: Berhahn Books. Recuperado de http://www.berghahnbooks.com/books/freepdfs/AndersonLevittAnth ropologies_intro.pdf

Ball, S., Maguire, M. \& Braun, A. (2012). How schools do policy: policy enactments in secondary schools. New York: Routledge.

Barbosa Moreira, A. F. (1990). Sociologia do currículo: origens, desenvolvimento e contribuições. Aberto, 46, 73-83. Recuperado de http://rbep.inep.gov.br/oj33/index.php/emaberto/article/view/2097

Batallán, G. (2007). Docentes de infancia. Antropología del trabajo en la escuela primaria. Buenos Aires: Paidós.

Bericat Alastuey, E. (2000) La sociología de la emoción y la emoción en la sociología. Papers Revista de Sociología, 62, 145-176. Recuperado de http://papers.uab.cat/article/view/v62-bericat/pdf-es

Berlant, L. (2011). El corazón de la nación. Ensayos sobre politica y sentimentalismo. México D. F.: Fondo de Cultura Económica.

Biesta, G. y Säfström, C. (2018). Un manifiesto por la educación. Praxis educativa, 22(2), 20-36.

Bowe, R., Ball, S. \& Gold, A. (1992). Reforming education \& changing schools: case studies in Policy Sociology. London: Routledge.

Cantero, G., Celman, S., Ulla de Costa, Z., Andretich, G., Altamirano, S. Grinóveno, N. y Martínez Oyhamburu, D. (2006). Reformas educativas y ciudadanía (aportes para pensar el contexto de una nueva Ley de educación). Ciencia, Docencia y Tecnología, XVII(33), 13-45.

Deleuze, G. (2014). El poder. Curso sobre Foucault. Tomo II. Buenos Aires: Editorial Cactus.

Denzin, N. K. \& Lincoln, Y. S. (2005). Introduction. The Discipline and Practice of Qualitative Research. En The Sage Handbook of Qualitative Research (pp. 1-13). Thousand Oaks: Sage Publications Inc.

Dussel, I. (2019a). La cultura material de la escolarización: reflexiones en torno a un giro historiográfico. Educar em Revista, 35 (76), pp. 13-29.

Dussel, I. (2019b). Historias de cavernas, pupitres y guardapolvos. Los aportes del giro material en la historia de la educación. En P. Pineau et. al.Latinoamérica: la educación y su historia. Nuevos enfoquespara su debate yenseñanza (pp. 35-55). Buenos Aires: Editorial de la Facultad de Filosofía y Letras, Universidad de Buenos Aires.

Feldfeber, M. y Gluz, N. (2011). Las políticas educativas en Argentina: herencias de los 90, contradicciones y tendencias de "nuevo signo". Educação \& Sociedade, 32(115), 339-356. Recuperado de http://www.cedes.unicamp.br.

Ferreyra, H. (Coord.). (2012). Aproximaciones a la educación secundaria en la Argentina 2000-2010: entramados, análisis y propuestas para el debate. Córdoba: Comunic-Arte.

Foucault, M. (1992). Microfísica del poder. Buenos Aires: Siglo XXI editores.

Foucault, M. (2002). La arqueología del saber. Buenos Aires: Siglo XXI editores.

Foucault, M. (2008). Historia de la sexualidad 1. La voluntad de saber. Buenos Aires: Siglo XXI. 
Frigerio, G. (2000). ¿Las reformas educativas reforman las escuelas o las escuelas reforman las reformas? Seminario sobre Prospectivas de la Educación en América Latina y el Caribe. Reunión de trabajo: Educación y Prospectiva. UNESCO - OREALC. Santiago de Chile, Chile. Recuperado de: https://www.oei.es/historico/reformaseduc ativas/reformas_educativas_reforman_escuelas_frigerio.pdf

Geertz, C. (2000). Géneros confusos. La refiguración del pensamiento social. En C. Reynoso (Comp.). El surgimiento de la antropología posmoderna (pp. 63-72). Barcelona: Gedisa.

Giovine, R. (2008). Nuevos escenarios, nuevas figuras y actores en el gobierno educativo: De la monopolización estatal a la pluralización de centros de regulación de las escuelas. V Jornadas de Sociología de la UNLP, 10, 11 y 12 de diciembre de 2008, La Plata, Argentina. Recuperado de http://www.memoria.fahce.unlp.edu.ar/trab_eventos/ ev.6096/ev.6096.pdf

Giovine, R. (2012). El arte de gobernar el sistema educativo. Discursos de Estado y redes de integración socioeducativas. Bernal: Quilmes Editorial.

Gleason, M. (2017). Metaphor, materiality, and method: the central role of embodiment in the history of education. Paedagogica Historica: International Journal of the History of Education. https://doi.org/10.1080/00309230.2 017.1355328

Goetz, J. P. y LeCompte, M. D. (1988). Etnografia y diseño cualitativo en investigación educativa. Madrid: Morata.

Gorostiaga, J. y Tello, C. (2011). Globalización y reforma educativa en América Latina: un análisis inter-textual. Revista Brasileira de Educação, 16(47), 363-368.

Grinberg, S. (2008). Educación y poder en el Siglo XXI. Gubernamentalidad y Pedagogía en las sociedades de gerenciamiento. Buenos Aires: Miño y Dávila.

Guber, R. (2005). El salvaje metropolitano. Buenos Aires: Paidós.

Hammersley, M. y Atkinson, P. (1994). ¿Qué es la etnografía? En Etnografia. Métodos de Investigación (pp. 1-21). Barcelona: Paidós.

Lacan, J. (1989). El tiempo lógico y el aserto de certidumbre anticipada. Un nuevo sofisma. En Escritos I. Buenos Aires: Siglo XXI Editores.

Lawn, M. \& Grosvenor, I. (1999). Imagining a Project: Networks, Discourses and Spaces - Towards a New Archaeology of Urban Education. Paedagogica Historica: International Journal of the History of Education, pp. 380-393. https://doi.org/ 10.1080/0030923990350205.

Lawn, M. \& Grosvenor, I. (2001). "When in doubt, preserve": exploring the traces of teaching and material culture in English schools. History of Education: Journal of the History of Education Society, pp. 117-127. https://doi.org/ $10.1080 / 00467600010012418$.

Marcus, G. (2013). Los legados de Writing Culture y el futuro cercano de la forma etnográfica: un boceto. Antípoda. Revista de Antropología y Arqueología, 16, 59-80.

Massumi, B. (2009). Interview: Of Microperception and Micropolitics. Inflexions: A Journal for Research-Creation, 3, 1-20. Recuperado de http://www.inflexions.org/n3_Of-Microperception-and-Micropolitics-An-Interviewwith-Brian-Massumi.pdf

Miranda, E. (2001). La Reforma Educativa en Argentina: Análisis político de su implementación en la provincia de Córdoba. Trabajo presentado en Reunión de Latin American Studies Asociation. LASA, Washington.

Miranda, E. y Lamfri, N. (2017). La educación secundaria. Cuando la política educativa llega a la escuela. Buenos Aires: Miño y Dávila.

Montes de Oca, L. (2015). Entre activistas, funcionarios e industriales. Aplicación de la etnografía -enfocada y política- en escenarios de gobernanza. Nueva Antropología, 28(83), 25-46. Recuperado de http://www.sci elo.org.mx/pdf/na/v28n83/v28n83a3.pdf

Pallma, S. y Sinisi, L. (2004). Tras las huellas de la etnografía educativa. Aportes para una reflexión. Cuadernos de Antropología Social, 19, 121-138.

Popkewitz, T. (1994). Sociología politica de las reformas educativas. Madrid: Morata. 
Rockwell, E. (1987). Reflexiones sobre el proceso etnográfico (1982-1985). En E. Rockwell y J. Ezpeleta (Coords.). Para observar la escuela, caminos y nociones (pp. 1-38). México D. F.: DIE.

Rockwell, E. (Coord.) (1995). La escuela cotidiana. México D. F.: Fondo de Cultura Económica.

Rockwell, E. y Anderson-Levitt, K. (2015). Importantes correntes de pesquisa etnográfica sobre educação: maiorias, minorias emigraçóes através das Américas. Educação e Pesquisa, 41(n. especial), 1129-1135. https://doi.org/ $10.1590 /$ S1517-9702201508148880

Rockwell, E. y Ezpeleta, J. (2007). A escola: relato de um processo inacabado de construção. Currículo sem Fronteiras, 7(2), 131-147.

Scribano, A. (2013). Sociología de los cuerpos/emociones. Revista Latinoamericana de Estudios sobre Cuerpos, Emociones y Sociedad, 4(10), 93 - 113. Recuperado de http://www.relaces.com.ar

Tiramonti, G. (2015a). La reforma de la escuela media: un anhelo frustrado. Propuesta Educativa, 2(44), 24-37.

Tiramonti, G. (2015b). Introducción. Escuela secundaria siglo XXI: un recorrido por algunas de sus reformas de cara a los desafíos de la sociedad contemporánea. Propuesta Educativa, 2(44), 6-7.

Villagran, C. (2016). Curriculum y regulación de la vida escolar: los procesos de reforma y los docentes. Un estudio en la escuela primaria y secundaria en la ciudad de Caleta Olivia. Tesis de Maestría. Universidad Nacional de la Patagonia Austral. Unidad Académica Caleta Olivia, Caleta Olivia, Santa Cruz, Argentina.

Villagran, C. (2018). Recepción y puesta en acto de la reforma del curriculum. Un estudio del devenir de la reforma en escuelas secundarias públicas de Santa Cruz. Tesis doctoral, Universidad Nacional de Buenos Aires, Buenos Aires.

Villagran, C. y Grinberg, S. (2019). Animarse a: políticas curriculares, retórica reformista y responsabilización. Educação \& Realidade, Porto Alegre, 44 (3), pp. 1-19. Disponible en: http://dx.doi.org/10.1590/2175-62368 3028

Youdell, D. (2010). Queer outings: uncomfortable stories about the subjects of post-structural school ethnography. International Journal of Qualitative Studies in Education, 23(1), 87-100. https://doi.org/ $10.1080 / 09518390903447168$

\section{Notas}

1 Proponemos la noción de afectos siguiendo la perspectiva del giro afectivo que sostiene que los afectos son construcciones sociales, producidas por otros y por el sujeto mismo; por ello, son siempre políticos. No nos referimos a los afectos en el sentido planteado por la sociología de las emociones (Bericat Alastuey, 2000; Scribano, 2013). Es posible reconocer un conjunto de investigaciones en torno a la sociología de las emociones y su crecimiento y presencia en eventos académicos de diferentes campos disciplinares. Nos distanciamos de estas perspectivas, desarrolladas a partir de los años ochenta, que entienden las emociones ligadas a estados psicológicos del ser sintiente.

2 Entendemos las reformas en el sentido planteado por Popkewitz (1994), esto es, como tecnologías de regulación y prácticas de gobierno. Por otra parte, se podría postular como equivalentes reformas y políticas educativas. Según Ball (2013), constituyen dos caras de una misma moneda. En este sentido, argumenta que de algún modo la política educativa siempre ha estado vinculada con la reforma, con el deseo de hacer las cosas de forma diferente, para cambiar aquello que ya está hecho. Asimismo, cada reforma se asienta en una política educativa e involucra a un conjunto de ellas. Sin embargo, no todas las políticas educativas en tanto políticas públicas implican una reforma estructural del sistema educativo, aunque sí generan cambios a nivel de las instituciones.

3 Para referirse a este nivel microfísico en el que el poder circula dotado de su omnipresencia e inmanencia en el campo político, Foucault (1992) habla de entidades moleculares, de células, de los puntos más pequeños de la sociedad, de las prácticas reales y efectivas. Deleuze (2014), por otro lado, en su analítica del poder refiere a la idea de corpúsculos sociales, al plano microfísico y micropolítico de las relaciones sociales y a las prácticas de ejercicio y circulación del poder.

4 Abu Lughod (1988) realiza un ejercicio más que interesante en este sentido respecto de su trabajo de campo con los beduinos árabes.

5 Parafraseamos a Lacan (1989) en su planteo sobre el tiempo lógico intersubjetivo. 